\title{
A necessary and sufficient condition for the strong convergence of nonexpansive mappings in Banach spaces
}

Shuang Wang ${ }^{1,2}$ and Dingbian Qian ${ }^{1 *}$

\section{"Correspondence:}

dbqian@suda.edu.cn

'School of Mathematical Sciences,

Soochow University, Suzhou,

215006, China

Full list of author information is

available at the end of the article

\begin{abstract}
In this paper, we establish a necessary and sufficient condition for the strong convergence of nonexpansive mappings in a uniformly convex and 2-uniformly smooth Banach space. It is worth pointing out that we remove some quite restrictive conditions in the corresponding results. An appropriate example, such that all conditions of this result are satisfied and that other conditions are not satisfied, is provided.
\end{abstract}

MSC: $47 \mathrm{H} 05 ; 47 \mathrm{H} 09 ; 47 \mathrm{H} 10$

Keywords: strong convergence; fixed points; nonexpansive mapping; $\boldsymbol{\eta}$-strongly accretive; 2-uniformly smooth Banach spaces

\section{Introduction}

Let $X$ be a real Banach space with the dual space $X^{*}$. The value of $f \in X^{*}$ at $x \in X$ is denoted by $\langle x, f\rangle$. The normal duality mapping $J$ from $X$ into a family of nonempty (by the HahnBanach theorem) weak-star compact subsets of $X^{*}$ is defined by

$$
J(x)=\left\{f \in X^{*}:\langle x, f\rangle=\|x\|^{2}=\|f\|^{2}\right\}, \quad \forall x \in X .
$$

Let $U=\{x \in X:\|x\|=1\}$. A Banach space $X$ is said to be uniformly convex if for each $\epsilon \in(0,2]$, there exists $\delta>0$ such that for any $x, y \in U$,

$$
\|x-y\| \geq \epsilon \quad \text { implies } \quad\left\|\frac{x+y}{2}\right\| \leq 1-\delta .
$$

It is known that a uniformly convex Banach space is reflexive and strictly convex. A Banach space $X$ is said to be smooth if the limit

$$
\lim _{t \rightarrow 0} \frac{\|x+t y\|-\|x\|}{t}
$$

exists for all $x, y \in U$. It is said to be uniformly smooth if limit (1.1) is attained uniformly for $x, y \in U$. It is well known that if $X$ is smooth, then $J$ is single-valued and continuous from the norm topology of $X$ to the weak-star topology of $X^{*}$, i.e., norm to weak* continuous. It is also well known that if $X$ is uniformly smooth, then $J$ is uniformly continuous on 
bounded subsets of $X$ from the strong topology of $X$ to the strong topology of $X^{*}$, i.e., uniformly norm-to-norm continuous on any bounded subset of $X$; see $[1,2]$ for more details.

Also, we define a function $\rho:[0, \infty) \rightarrow[0, \infty)$ called the modulus of smoothness of $X$ as follows:

$$
\rho(\tau)=\sup \left\{\frac{1}{2}(\|x+y\|+\|x-y\|)-1: x, y \in X,\|x\|=1,\|y\|=\tau\right\} .
$$

It is known that $X$ is uniformly smooth if and only if $\lim _{\tau \rightarrow 0} \rho(\tau) / \tau=0$. Let $q$ be a fixed real number with $1<q \leq 2$. Then a Banach space $X$ is said to be $q$-uniformly smooth if there exists a constant $c>0$ such that $\rho(\tau) \leq c \tau^{q}$ for all $\tau>0$. One should note that no Banach space is $q$-uniformly smooth for $q>2$; see [3] for more details. So, in this paper, we focus on a 2-uniformly smooth Banach space. It is well known that Hilbert spaces and Lebesgue $L^{p}(p \geq 2)$ spaces are uniformly convex and 2-uniformly smooth.

Recall that a mapping $T: X \rightarrow X$ is said to be nonexpansive if

$$
\|T x-T y\| \leq\|x-y\|, \quad \forall x, y \in X .
$$

A point $x \in X$ is a fixed point of $T$ if $T x=x$. Let $\operatorname{Fix}(T)$ denote the set of fixed points of $T$; that is, $\operatorname{Fix}(T)=\{x \in X: T x=x\}$.

A mapping $\bar{f}: X \rightarrow X$ is called strongly pseudo-contractive if there exists a constant $\rho \in(0,1)$ and $j(x-y) \in J(x-y)$ satisfying

$$
\langle\bar{f}(x)-\bar{f}(y), j(x-y)\rangle \leq \rho\|x-y\|^{2}, \quad \forall x, y \in X .
$$

A mapping $f: X \rightarrow X$ is a contraction if there exists a constant $\alpha \in(0,1)$ such that

$$
\|f(x)-f(y)\| \leq \alpha\|x-y\|, \quad \forall x, y \in X .
$$

Since $\langle f(x)-f(y), j(x-y)\rangle \leq\|f(x)-f(y)\|\|x-y\| \leq \alpha\|x-y\|^{2}$, we have that $f$ is a strong pseudo-contraction.

Let $\eta>0$, a mapping $\bar{F}$ of $X$ into $X$ is said to be $\eta$-strongly accretive if there exists $j(x-y) \in$ $J(x-y)$ such that

$$
\langle\bar{F} x-\bar{F} y, j(x-y)\rangle \geq \eta\|x-y\|^{2}
$$

for all $x, y \in X$. A mapping $\bar{F}$ of $X$ into $X$ is said to be $k$-Lipschitzian if, for $k>0$,

$$
\|\bar{F} x-\bar{F} y\| \leq k\|x-y\|
$$

for all $x, y \in X$. It is well known that if $X$ is a Hilbert space, then an $\eta$-strongly accretive operator coincides with an $\eta$-strongly monotone operator.

Yamada [4] introduced the following hybrid iterative method for solving the variational inequality in a Hilbert space:

$$
x_{n+1}=T x_{n}-\mu \lambda_{n} F\left(T x_{n}\right), \quad n \geq 0,
$$


where $F$ is a $k$-Lipschitzian and $\eta$-strongly monotone operator with $k>0, \eta>0$ and $0<$ $\mu<2 \eta / k^{2}$. Let a sequence $\left\{\lambda_{n}\right\}$ of real numbers in $(0,1)$ satisfy the conditions below:

(A1) $\lim _{n \rightarrow \infty} \lambda_{n}=0$,

(A2) $\sum_{n=0}^{\infty} \lambda_{n}=\infty$,

(A3) $\lim _{n \rightarrow \infty}\left(\lambda_{n}-\lambda_{n+1}\right) / \lambda_{n+1}^{2}=0$.

He proved that $\left\{x_{n}\right\}$ generated by (1.2) converges strongly to the unique solution of the variational inequality

$$
\langle F \tilde{x}, x-\tilde{x}\rangle \geq 0, \quad \forall x \in \operatorname{Fix}(T) .
$$

An example of sequence $\left\{\lambda_{n}\right\}$ which satisfies conditions (A1)-(A3) is given by $\lambda_{n}=1 / n^{\sigma}$, where $0<\sigma<1$. We note that condition (A3) was first used by Lions [5]. It was observed that Lion's conditions on the sequence $\left\{\lambda_{n}\right\}$ excluded the canonical choice $\lambda_{n}=1 / n$. This was overcome in 2003 by $\mathrm{Xu}$ and Kim [6] in a Hilbert space. They proved that if $\left\{\lambda_{n}\right\}$ satisfies conditions (A1), (A2) and (A4)

(A4) $\lim _{n \rightarrow \infty} \lambda_{n} / \lambda_{n+1}=1$ or, equivalently, $\lim _{n \rightarrow \infty}\left(\lambda_{n}-\lambda_{n+1}\right) / \lambda_{n+1}=0$,

then $\left\{x_{n}\right\}$ is strongly convergent to the unique solution $u^{*}$ of the variational inequality $\left\langle F u^{*}, v-u^{*}\right\rangle \geq 0, \forall v \in C$. It is easy to see that condition (A4) is strictly weaker than condition (A3), coupled with conditions (A1) and (A2). Moreover, (A4) includes the important and natural choice $\{1 / n\}$ of $\left\{\lambda_{n}\right\}$.

In 2010, Tian [7] improved Yamada's method (1.2) and established the following strong convergence theorems.

Theorem 1.1 ([7, Theorem 3.1]) Let $H$ be a Hilbert space. Let $T: H \rightarrow H$ be a nonexpansive mapping with $\operatorname{Fix}(T) \neq \emptyset$, and let $f: H \rightarrow H$ be a contraction mapping with $\alpha \in(0,1)$. Assume that $\left\{x_{t}\right\}$ is defined by

$$
x_{t}=t \gamma f\left(x_{t}\right)+(I-t \mu F) T x_{t}
$$

where $F$ is a $k$-Lipschitzian and $\eta$-strongly monotone operator on a Hilbert space $H$ with $k>0, \eta>0$. Let $0<\mu<2 \eta / k^{2}, 0<\gamma<\mu\left(\eta-\frac{\mu k^{2}}{2}\right) / \alpha=\tau / \alpha$ and $0<t<1$. Then $x_{t}$ converges strongly as $t \rightarrow 0$ to a fixed point $\tilde{x}$ of $T$, which solves the variational inequality $\langle(\mu F-$ $\gamma f) \tilde{x}, \tilde{x}-z\rangle \leq 0, z \in \operatorname{Fix}(T)$.

Theorem 1.2 ([7, Theorem 3.1]) Let $H$ be a Hilbert space. Let $T: H \rightarrow H$ be a nonexpansive mapping with $\operatorname{Fix}(T) \neq \emptyset$, let $f: H \rightarrow H$ be a contraction mapping with $\alpha \in(0,1)$, and let $F$ be a $k$-Lipschitzian and $\eta$-strongly monotone operator on $H$ with $k>0, \eta>0$ and $0<\mu<2 \eta / k^{2}$. For an arbitrary $x_{0} \in H$, let $\left\{x_{n}\right\}$ be generated by

$$
x_{n+1}=\left(I-\alpha_{n} \mu F\right) T x_{n}+\alpha_{n} \gamma f\left(x_{n}\right), \quad n \geq 0,
$$

where $0<\gamma<\mu\left(\eta-\frac{\mu k^{2}}{2}\right) / \alpha=\tau / \alpha$ and $\left\{\alpha_{n}\right\} \subset(0,1)$ satisfies

(C1) $\alpha_{n} \rightarrow 0$, 
(C2) $\sum_{n=0}^{\infty} \alpha_{n}=\infty$

(C3) either $\sum_{n=0}^{\infty}\left|\alpha_{n+1}-\alpha_{n}\right|<\infty$ or $\lim _{n \rightarrow \infty} \frac{\alpha_{n+1}}{\alpha_{n}}=1$.

Then $\left\{x_{n}\right\}$ converges strongly to $\tilde{x}$ that is obtained in Theorem 1.1.

We remind the reader of the following facts: (i) The results are obtained when the underlying space is a Hilbert space in Yamada [4], Xu [6] and Tian [7]. (ii) In order to guarantee the strong convergence of the iterative sequence $\left\{x_{n}\right\}$, there is at least one parameter sequence converging to zero (i.e., $\alpha_{n} \rightarrow \infty$ or $\lambda_{n} \rightarrow 0$ ) in Yamada [4], Xu [6] and Tian [7]. (iii) The parameter sequence satisfies the condition $\lim _{n \rightarrow \infty} \lambda_{n} / \lambda_{n+1}=1$ (or $\left.\lim _{n \rightarrow \infty} \alpha_{n+1} / \alpha_{n}=1\right)$.

In this paper, we establish a necessary and sufficient condition for the strong convergence of $\left\{x_{n}\right\}$ generated by (3.7) (defined below) in a uniformly convex and 2-uniformly smooth Banach space. In the meantime, we remove the control condition $(\mathrm{C} 1)$ and replace condition (C3) with (C3') (defined below) in the result of Tian [7]. It is worth pointing out that we use a new method to prove our main results. The results presented in this paper can be viewed as an improvement, supplement and extension of the results obtained in $[4,6,7]$

\section{Preliminaries}

For the sequence $\left\{x_{n}\right\}$ in $X$, we write $x_{n} \rightarrow x$ to indicate that the sequence $\left\{x_{n}\right\}$ converges weakly to $x . x_{n} \rightarrow x$ means that $\left\{x_{n}\right\}$ converges strongly to $x$. In order to prove our main results, we need the following lemmas.

Lemma 2.1 ([8]) Let $q$ be a given real number with $1<q \leq 2$, and let $X$ be a q-uniformly smooth Banach space. Then

$$
\left.\|x+y\|^{q} \leq\|x\|^{q}+q \mid y, J_{q}(x)\right)+2\|K y\|^{q}
$$

for all $x, y \in X$, where $K$ is a q-uniformly smooth constant of $X$ and $J_{q}$ is the generalized duality mapping from $X$ into $2^{X^{*}}$ defined by

$$
J_{q}(x)=\left\{f \in X^{*}:\langle x, f\rangle=\|x\|^{q},\|f\|=\|x\|^{q-1}\right\}
$$

for all $x \in X$.

Lemma 2.2 ([2]) Let $C$ be a nonempty bounded closed convex subset of a uniformly convex Banach space $X$, and let $T$ be a nonexpansive mapping of $C$ into itself. If $\left\{x_{n}\right\}$ is a sequence of $C$ such that $x_{n} \rightarrow x$ and $x_{n}-T x_{n} \rightarrow 0$, then $x$ is a fixed point of $T$.

Lemma 2.3 $([9,10])$ Let $\left\{s_{n}\right\}$ be a sequence of nonnegative real numbers satisfying

$$
s_{n+1} \leq\left(1-\lambda_{n}\right) s_{n}+\lambda_{n} \delta_{n}+\gamma_{n}, \quad n \geq 0,
$$

where $\left\{\lambda_{n}\right\},\left\{\delta_{n}\right\}$ and $\left\{\gamma_{n}\right\}$ satisfy the following conditions: (i) $\left\{\lambda_{n}\right\} \subset[0,1]$ and $\sum_{n=0}^{\infty} \lambda_{n}=\infty$, (ii) $\lim \sup _{n \rightarrow \infty} \delta_{n} \leq 0$ or $\sum_{n=0}^{\infty} \lambda_{n} \delta_{n}<\infty$, (iii) $\gamma_{n} \geq 0 \quad(n \geq 0), \sum_{n=0}^{\infty} \gamma_{n}<\infty$. Then $\lim _{n \rightarrow \infty} s_{n}=0$. 
The following lemma is easy to prove.

Lemma 2.4 Let $X$ be a Banach space, let $\bar{f}: X \rightarrow X$ be a strongly pseudo-contractive operator with $0<\rho<1$, and let $\bar{F}: X \rightarrow X$ be a $k$-Lipschitzian and $\eta$-strongly accretive operator with $k>0, \eta>0$. Then, for $\rho<\mu \eta$,

$$
\langle(\mu \bar{F}-\bar{f}) x-(\mu \bar{F}-\bar{f}) y, j(x-y)\rangle \geq(\mu \eta-\rho)\|x-y\|^{2}, \quad \forall x, y \in X
$$

that is, $\mu \bar{F}-\bar{f}$ is a strongly accretive operator with coefficient $\mu \eta-\rho$.

Lemma 2.5 Let $X$ be a real 2-uniformly smooth Banach space. Let t be a number in $(0,1)$, and let $\mu>0$. Let $\bar{F}: X \rightarrow X$ be an operator such that, for some constant $0<\eta \leq \sqrt{2} k K$, $\bar{F}$ is $k$-Lipschitzian and $\eta$-strongly accretive. Then $S=(I-t \mu \bar{F}): X \rightarrow X$ is a contraction provided $\mu \leq \eta /\left(2 k^{2} K^{2}\right)$, that is,

$$
\|S x-S y\| \leq(1-t \tau)\|x-y\|, \quad \forall x, y \in X,
$$

where $\tau=1-\sqrt{1-2 \mu \eta+2 \mu^{2} k^{2} K^{2}} \in(0,1]$.

Proof Using Lemma 2.1, we have

$$
\begin{aligned}
\|(I-\mu \bar{F}) x-(I-\mu \bar{F}) y\|^{2} & \leq\|x-y\|^{2}-2 \mu\langle\bar{F} x-\bar{F} y, j(x-y)\rangle+2 \mu^{2} K^{2}\|\bar{F} x-\bar{F} y\|^{2} \\
& \leq\|x-y\|^{2}-2 \mu \eta\|x-y\|^{2}+2 \mu^{2} K^{2} k^{2}\|x-y\|^{2} \\
& =\left(1-2 \mu \eta+2 \mu^{2} k^{2} K^{2}\right)\|x-y\|^{2} .
\end{aligned}
$$

It follows from $0<\eta \leq \sqrt{2} k K$ and $0<\mu \leq \eta /\left(2 k^{2} K^{2}\right)$ that

$$
\|(I-\mu \bar{F}) x-(I-\mu \bar{F}) y\| \leq \sqrt{1-2 \mu \eta+2 \mu^{2} k^{2} K^{2}}\|x-y\|
$$

for all $x, y \in X$. Therefore, we have

$$
\begin{aligned}
\|S x-S y\| & =\|(1-t)(x-y)-t[(I-\mu \bar{F}) y-(I-\mu \bar{F}) x]\| \\
& \leq(1-t)\|x-y\|+t\|(I-\mu \bar{F}) x-(I-\mu \bar{F}) y\| \\
& \leq(1-t)\|x-y\|+t \sqrt{1-2 \mu \eta+2 \mu^{2} k^{2} K^{2}}\|x-y\| \\
& =(1-t \tau)\|x-y\|,
\end{aligned}
$$

where $\tau=1-\sqrt{1-2 \mu \eta+2 \mu^{2} k^{2} K^{2}} \in(0,1]$.

\section{Main results}

Throughout this section, let $X$ be a uniformly convex and 2-uniformly smooth Banach space. Let $T: X \rightarrow X$ be a nonexpansive mapping with $\operatorname{Fix}(T) \neq \emptyset$. Let $\bar{F}: X \rightarrow X$ be a $k$ Lipschitzian and $\eta$-strongly accretive operator with $0<\eta \leq \sqrt{2} k K$. Let $\mu \in\left(0, \eta /\left(2 k^{2} K^{2}\right)\right]$ and $\tau=1-\sqrt{1-2 \mu \eta+2 \mu^{2} k^{2} K^{2}}$. Let $\bar{f}: X \rightarrow X$ be a Lipschitzian and strongly pseudocontractive operator with $0<\rho<\tau$. Let $t$ be a number in $(0,1)$. Consider a mapping $S_{t}$ on 
$X$ defined by

$$
S_{t} x=t \bar{f}(x)+(I-t \mu \bar{F}) T x, \quad x \in X .
$$

It is easy to see that the mapping $S_{t}$ is strongly pseudo-contractive. Indeed, from Lemma 2.5, we have

$$
\begin{aligned}
\left\langle S_{t} x-S_{t} y, j(x-y)\right\rangle & =t\langle\bar{f}(x)-\bar{f}(y), j(x-y)\rangle+\langle(I-t \mu \bar{F}) T x-(I-t \mu \bar{F}) T y, j(x-y)\rangle \\
& \leq t \rho\|x-y\|^{2}+\|(I-t \mu \bar{F}) T x-(I-t \mu \bar{F}) T y\|\|x-y\| \\
& \leq t \rho\|x-y\|^{2}+(1-t \tau)\|x-y\|^{2} \\
& \leq[1-t(\tau-\rho)]\|x-y\|^{2}
\end{aligned}
$$

for all $x, y \in X$. Since $\bar{f}$ is Lipschitzian and $I-t \mu \bar{F}$ is contractive, hence $S_{t}$ is continuous. So, by Deimling [11], we can obtain that $S_{t}$ has a unique fixed point which we denoted by $x_{t}$, that is,

$$
x_{t}=t \bar{f}\left(x_{t}\right)+(I-t \mu \bar{F}) T x_{t}, \quad x_{t} \in X .
$$

Our first main result below shows that $\left\{x_{t}\right\}$ converges strongly as $t \rightarrow 0^{+}$to a fixed point of $T$ which solves some variational inequality.

Theorem $3.1\left\{x_{t}\right\}$ generated by the implicit method (3.1) converges in norm as $t \rightarrow 0^{+}$to the unique solution $x^{*} \in \operatorname{Fix}(T)$ of the variational inequality

$$
\left\langle(\mu \bar{F}-\bar{f}) x^{*}, j\left(x^{*}-u\right)\right\rangle \leq 0, \quad u \in \operatorname{Fix}(T) .
$$

Proof It is easy to see the uniqueness of a solution of variational inequality (3.2). By Lemma $2.4, \mu \bar{F}-\bar{f}$ is strongly accretive, so variational inequality (3.2) has only one solution. Below we use $x^{*} \in \operatorname{Fix}(T)$ to denote the unique solution of (3.2).

Next, we prove that $\left\{x_{t}\right\}$ is bounded. Take $u \in \operatorname{Fix}(T)$, from (3.1) and using Lemma 2.5, we have

$$
\begin{aligned}
\left\|x_{t}-u\right\|^{2}= & \left\langle x_{t}-u, j\left(x_{t}-u\right)\right\rangle \\
= & t\left\langle\bar{f}\left(x_{t}\right)-\bar{f}(u), j\left(x_{t}-u\right)\right\rangle+\left\langle(I-t \mu \bar{F}) T x_{t}-(I-t \mu \bar{F}) T u, j\left(x_{t}-u\right)\right\rangle \\
& \left.+t \bar{f}(u)-\mu \bar{F} u, j\left(x_{t}-u\right)\right\rangle \\
\leq & t \rho\left\|x_{t}-u\right\|^{2}+\left\|(I-t \mu \bar{F}) T x_{t}-(I-t \mu \bar{F}) T u\right\|\left\|x_{t}-u\right\| \\
& +t\|\bar{f}(u)-\mu \bar{F} u\|\left\|x_{t}-u\right\| \\
\leq & {[1-t(\tau-\rho)]\left\|x_{t}-u\right\|^{2}+t\|\bar{f}(u)-\mu \bar{F} u\|\left\|x_{t}-u\right\| . }
\end{aligned}
$$

It follows that

$$
\left\|x_{t}-u\right\| \leq \frac{1}{\tau-\rho}\|\bar{f}(u)-\mu \bar{F} u\| .
$$

Therefore, $\left\{x_{t}\right\}$ is bounded, and so are the nets $\left\{\bar{f}\left(x_{t}\right)\right\}$ and $\left\{\bar{F} T x_{t}\right\}$. 
On the other hand, from (3.1) we obtain

$$
\left\|x_{t}-T x_{t}\right\|=t\left\|\bar{f}\left(x_{t}\right)-\mu \bar{F} T x_{t}\right\| \rightarrow 0 \quad\left(t \rightarrow 0^{+}\right) .
$$

Next, we show that $\left\{x_{t}\right\}$ is relatively norm-compact as $t \rightarrow 0^{+}$. Assume that $\left\{t_{n}\right\} \in(0,1)$ such that $t_{n} \rightarrow 0^{+}$as $n \rightarrow \infty$. Put $x_{n}:=x_{t_{n}}$. It follows from (3.3) that

$$
\left\|x_{n}-T x_{n}\right\| \rightarrow 0 \quad(n \rightarrow \infty) .
$$

For a given $u \in \operatorname{Fix}(T)$, by (3.1) and using Lemma 2.5, we have

$$
\begin{aligned}
\left\|x_{t}-u\right\|^{2}= & \left\langle x_{t}-u, j\left(x_{t}-u\right)\right\rangle \\
= & t\left\langle\bar{f}\left(x_{t}\right)-\bar{f}(u), j\left(x_{t}-u\right)\right\rangle+\left\langle(I-t \mu \bar{F}) T x_{t}-(I-t \mu \bar{F}) T u, j\left(x_{t}-u\right)\right\rangle \\
& +t\left|\bar{f}(u)-\mu \bar{F} u, j\left(x_{t}-u\right)\right\rangle \\
\leq & {[1-t(\tau-\rho)]\left\|x_{t}-u\right\|^{2}+t\left|\bar{f}(u)-\mu \bar{F} u, j\left(x_{t}-u\right)\right\rangle, }
\end{aligned}
$$

that is,

$$
\left\|x_{t}-u\right\|^{2} \leq \frac{1}{\tau-\rho}\left\langle\bar{f}(u)-\mu \bar{F} u, j\left(x_{t}-u\right)\right\rangle .
$$

In particular,

$$
\left\|x_{n}-u\right\|^{2} \leq \frac{1}{\tau-\rho}\left\langle\bar{f}(u)-\mu \bar{F} u, j\left(x_{n}-u\right)\right\rangle .
$$

Since $\left\{x_{t}\right\}$ is bounded, without loss of generality, we may assume that $\left\{x_{n}\right\}$ converges weakly to a point $\tilde{x}$. By (3.4) and using Lemma 2.2, we have $\tilde{x} \in \operatorname{Fix}(T)$. Then by (3.5), $x_{n} \rightarrow \tilde{x}$. This has proved the relative norm compactness of the net $\left\{x_{t}\right\}$ as $t \rightarrow 0^{+}$.

We next show that $\tilde{x}$ solves variational inequality (3.2). Observe that

$$
(\mu \bar{F}-\bar{f})\left(x_{t}\right)=-\frac{1}{t}(I-T) x_{t}+\mu\left(\bar{F} x_{t}-\bar{F} T x_{t}\right) .
$$

Since $I-T$ is accretive (this is due to the nonexpansiveness of $T$ ), for any $u \in \operatorname{Fix}(T)$, we can deduce immediately that

$$
\left\langle(I-T) x_{t}-(I-T) u, j\left(x_{t}-u\right)\right\rangle \geq 0 .
$$

Therefore, for any $u \in \operatorname{Fix}(T)$

$$
\begin{aligned}
\left\langle(\mu \bar{F}-\bar{f})\left(x_{t}\right), j\left(x_{t}-u\right)\right\rangle & =-\frac{1}{t}\left\langle(I-T) x_{t}, j\left(x_{t}-u\right)\right\rangle+\mu\left\langle\bar{F} x_{t}-\bar{F} T x_{t}, j\left(x_{t}-u\right)\right\rangle \\
& =-\frac{1}{t}\left\langle(I-T) x_{t}-(I-T) u, j\left(x_{t}-u\right)\right\rangle+\mu\left\langle\bar{F} x_{t}-\bar{F} T x_{t}, j\left(x_{t}-u\right)\right\rangle \\
& \leq \mu\left\langle\bar{F} x_{t}-\bar{F} T x_{t}, j\left(x_{t}-u\right)\right\rangle .
\end{aligned}
$$


Now, replace $t$ in (3.6) with $t_{n}$. Noting that $\bar{F} x_{t_{n}}-\bar{F} T x_{t_{n}} \rightarrow \bar{F} \tilde{x}-\bar{F} \tilde{x}=0$ for $\tilde{x} \in \operatorname{Fix}(T)$ as $n \rightarrow \infty$, we have

$$
\langle(\mu \bar{F}-\bar{f}) \tilde{x}, j(\tilde{x}-u)\rangle \leq 0 .
$$

That is $\tilde{x} \in \operatorname{Fix}(T)$ is a solution of (3.2), hence $\tilde{x}=x^{*}$ by uniqueness. In summary, we have shown that each cluster point of $\left\{x_{t}\right\}$ (as $t \rightarrow 0^{+}$) equals $x^{*}$. Therefore, $x_{t} \rightarrow x^{*}$ as $t \rightarrow 0^{+}$.

Remark 3.2 Compared with Theorem 3.1 of Tian [7], our Theorem 3.1 improves and extends Theorem 3.1 of Tian [7] in the following aspects:

(i) The framework of a Hilbert space is extended to a uniformly convex and 2-uniformly smooth Banach space.

(ii) The $\eta$-strongly monotone operator $F$ is extended to the case of an $\eta$-strongly accretive operator $\bar{F}$. The contraction $f: H \rightarrow H$ is extended to the case of a Lipschitzian and strongly pseudo-contractive operator $\bar{f}: X \rightarrow X$.

(iii) If we put $X=H, \bar{F}=F$ and $\bar{f}=\gamma f$, then our Theorem 3.1 reduces to Theorem 3.1 of Tian [7]. Thus, our Theorem 3.1 covers Theorem 3.1 of Tian [7] as a special case.

Next we consider the following iteration process: the initial guess $x_{0}$ is selected in $X$ arbitrarily and the $(n+1)$ th iterate $x_{n+1}$ is defined by

$$
x_{n+1}=\left(I-\alpha_{n} \mu \bar{F}\right) T x_{n}+\alpha_{n} \gamma f\left(x_{n}\right), \quad n \geq 0,
$$

where $f: X \rightarrow X$ is a contractive mapping with $0<\gamma \alpha<\tau,\left\{\alpha_{n}\right\}$ is a sequence in $(0,1)$ satisfying conditions $(\mathrm{C} 2)$ and

$\left(\mathrm{C} 3^{\prime}\right)\left|\alpha_{n+1}-\alpha_{n}\right| \leq o\left(\alpha_{n+1}\right)+\sigma_{n}$ with $\sigma_{n} \geq 0$ and $\sum_{n=0}^{\infty} \sigma_{n}<\infty$.

Besides the basic condition (C2) on the sequence $\alpha_{n}$, we have the control condition $\left(\mathrm{C}^{\prime}\right)$. It can obviously be replaced by one of the following:

(C3-1) $\sum_{n=0}^{\infty}\left|\alpha_{n+1}-\alpha_{n}\right|<\infty$;

(C3-2) $\lim _{n \rightarrow \infty} \alpha_{n+1} / \alpha_{n}=1$.

Indeed, (C3-1) implies (C3') by choosing $\sigma_{n}=\left|\alpha_{n+1}-\alpha_{n}\right|$, and (C3-2) implies (C3') by choosing $\sigma_{n}=0$. In this sense $\left(\mathrm{C}^{\prime}\right)$ is a weaker condition than the previous condition (C3).

Our second main result below shows that we have established a necessary and sufficient condition for the strong convergence of nonexpansive mappings in a uniformly convex and 2-uniformly smooth Banach space.

Theorem 3.3 Let $\left\{x_{n}\right\}$ be generated by algorithm (3.7) with the sequence $\alpha_{n}$ of parameters satisfying conditions $(\mathrm{C} 2)$ and $\left(\mathrm{C}^{\prime}\right)$. Then

$$
x_{n} \rightarrow x^{*} \quad \Leftrightarrow \quad \alpha_{n}\left(\gamma f\left(x_{n}\right)-\mu \bar{F} T x_{n}\right) \rightarrow 0 \quad(n \rightarrow \infty),
$$

where $x^{*} \in \operatorname{Fix}(T)$ solves the variational inequality $\left\langle(\mu \bar{F}-\gamma f) x^{*}, j\left(x^{*}-u\right)\right\rangle \leq 0, u \in \operatorname{Fix}(T)$. 
Proof On the one hand, suppose that $\alpha_{n}\left(\gamma f\left(x_{n}\right)-\mu \bar{F} T x_{n}\right) \rightarrow 0(n \rightarrow \infty)$. We proceed with the following steps.

Step 1. We claim that $\left\{x_{n}\right\}$ is bounded. In fact, taking $u \in \operatorname{Fix}(T)$, from (3.7) and using Lemma 2.5, we have

$$
\begin{aligned}
\left\|x_{n+1}-u\right\|= & \| \alpha_{n}\left(\gamma f\left(x_{n}\right)-\gamma f(u)\right)+\left(I-\alpha_{n} \mu \bar{F}\right) T x_{n} \\
& -\left(I-\alpha_{n} \mu \bar{F}\right) T u+\alpha_{n}(\gamma f(u)-\mu \bar{F} u) \| \\
\leq & \alpha_{n} \gamma \alpha\left\|x_{n}-u\right\|+\left(1-\alpha_{n} \tau\right)\left\|x_{n}-u\right\|+\alpha_{n}\|\gamma f(u)-\mu \bar{F} u\| \\
= & {\left[1-\alpha_{n}(\tau-\gamma \alpha)\right]\left\|x_{n}-u\right\|+\alpha_{n}(\tau-\gamma \alpha) \frac{\|\gamma f(u)-\mu \bar{F} u\|}{\tau-\gamma \alpha} } \\
\leq & \max \left\{\left\|x_{n}-u\right\|, \frac{\|\gamma f(u)-\mu \bar{F} u\|}{\tau-\gamma \alpha}\right\} .
\end{aligned}
$$

By induction, we have

$$
\left\|x_{n}-u\right\| \leq \max \left\{\left\|x_{0}-u\right\|, \frac{\|\gamma f(u)-\mu \bar{F} u\|}{\tau-\gamma \alpha}\right\}
$$

Therefore, $\left\{x_{n}\right\}$ is bounded. We also obtain that $\left\{f\left(x_{n}\right)\right\}$ and $\left\{\bar{F} T x_{n}\right\}$ are bounded.

Step 2. We claim that $\lim _{n \rightarrow \infty}\left\|x_{n+1}-x_{n}\right\|=0$. Observe that

$$
\begin{aligned}
\left\|x_{n+1}-x_{n}\right\|= & \| \alpha_{n} \gamma\left(f\left(x_{n}\right)-f\left(x_{n-1}\right)\right)+\gamma\left(\alpha_{n}-\alpha_{n-1}\right) f\left(x_{n-1}\right)+\left(I-\alpha_{n} \mu \bar{F}\right) T x_{n} \\
& -\left(I-\alpha_{n} \mu \bar{F}\right) T x_{n-1}+\left(\alpha_{n-1}-\alpha_{n}\right) \mu \bar{F} T x_{n-1} \| \\
\leq & {\left[1-\alpha_{n}(\tau-\gamma \alpha)\right]\left\|x_{n}-x_{n-1}\right\|+M\left|\alpha_{n}-\alpha_{n-1}\right| } \\
\leq & {\left[1-\alpha_{n}(\tau-\gamma \alpha)\right]\left\|x_{n}-x_{n-1}\right\|+M o\left(\alpha_{n}\right)+M \sigma_{n-1}, }
\end{aligned}
$$

where $M=\max \left\{\gamma f\left(x_{n-1}\right), \mu \bar{F} T x_{n-1}\right\}$. By Lemma 2.3, we have $\lim _{n \rightarrow \infty}\left\|x_{n+1}-x_{n}\right\|=0$.

Step 3. We claim that $\lim _{n \rightarrow \infty}\left\|x_{n}-T x_{n}\right\|=0$. Indeed, from Step 2, we have

$$
\begin{aligned}
\left\|x_{n}-T x_{n}\right\| & \leq\left\|x_{n}-x_{n+1}\right\|+\left\|x_{n+1}-T x_{n}\right\| \\
& =\left\|x_{n}-x_{n+1}\right\|+\left\|\alpha_{n}\left(\gamma f\left(x_{n}\right)-\mu \bar{F} T x_{n}\right)\right\| \rightarrow 0 \quad(n \rightarrow \infty) .
\end{aligned}
$$

Step 4. We claim that $\limsup _{n \rightarrow \infty}\left\langle(\gamma f-\mu \bar{F}) x^{*}, j\left(x_{n+1}-x^{*}\right)\right\rangle \leq 0$, where $x^{*}=\lim _{t \rightarrow 0^{+}} x_{t}$ and $x_{t}$ is defined by $x_{t}=t \gamma f\left(x_{t}\right)+(I-t \mu \bar{F}) T x_{t}$. Since $\left\{x_{n+1}\right\}$ is bounded, there exists a subsequence $\left\{x_{\{n+1\}_{k}}\right\}$ of $\left\{x_{n+1}\right\}$ which converges weakly to $\omega$. From Step 3, we obtain $T x_{\{n+1\}_{k}} \rightarrow \omega$. From Lemma 2.2, we have $\omega \in \operatorname{Fix}(T)$. Since $f$ is a contractive mapping, we have that $\gamma f$ is a Lipschitzian and strongly pseudo-contractive operator with $\gamma \alpha \in(0, \tau)$. Hence, using Theorem 3.1, we have $x^{*} \in \operatorname{Fix}(T)$ and

$$
\begin{aligned}
\limsup _{n \rightarrow \infty}\left\langle(\gamma f-\mu \bar{F}) x^{*}, j\left(x_{n+1}-x^{*}\right)\right\rangle & =\lim _{k \rightarrow \infty}\left\langle(\gamma f-\mu \bar{F}) x^{*}, j\left(x_{\{n+1\}_{k}}-x^{*}\right)\right\rangle \\
& =\left\langle(\gamma f-\mu \bar{F}) x^{*}, j\left(\omega-x^{*}\right)\right\rangle \leq 0 .
\end{aligned}
$$


Step 5. We claim that $\left\{x_{n}\right\}$ converges strongly to $x^{*} \in \operatorname{Fix}(T)$. From (3.7) and using Lemma 2.5, we have

$$
\begin{aligned}
\left\|x_{n+1}-x^{*}\right\|^{2}= & \left\langle\left(I-\alpha_{n} \mu \bar{F}\right) T x_{n}+\alpha_{n} \gamma f\left(x_{n}\right)-x^{*}, j\left(x_{n+1}-x^{*}\right)\right\rangle \\
= & \left\langle\left(I-\alpha_{n} \mu \bar{F}\right) T x_{n}-\left(I-\alpha_{n} \mu \bar{F}\right) T x^{*}, j\left(x_{n+1}-x^{*}\right)\right\rangle \\
& +\alpha_{n}\left\langle\gamma f\left(x_{n}\right)-\mu \bar{F} x^{*}, j\left(x_{n+1}-x^{*}\right)\right\rangle \\
\leq & \left(1-\alpha_{n} \tau\right)\left\|x_{n}-x^{*}\right\|\left\|x_{n+1}-x^{*}\right\|+\alpha_{n} \gamma \alpha\left\|x_{n}-x^{*}\right\|\left\|x_{n+1}-x^{*}\right\| \\
& +\alpha_{n}\left\langle\gamma f\left(x^{*}\right)-\mu \bar{F} x^{*}, j\left(x_{n+1}-x^{*}\right)\right\rangle \\
\leq & {\left[1-\alpha_{n}(\tau-\gamma \alpha)\right]\left\|x_{n}-x^{*}\right\|\left\|x_{n+1}-x^{*}\right\|+\alpha_{n}\left\langle\gamma f\left(x^{*}\right)-\mu \bar{F} x^{*}, j\left(x_{n+1}-x^{*}\right)\right\rangle } \\
\leq & \frac{\left[1-\alpha_{n}(\tau-\gamma \alpha)\right]^{2}}{2}\left\|x_{n}-x^{*}\right\|^{2}+\frac{1}{2}\left\|x_{n+1}-x^{*}\right\|^{2} \\
& +\alpha_{n}\left\langle\gamma f\left(x^{*}\right)-\mu \bar{F} x^{*}, j\left(x_{n+1}-x^{*}\right)\right\rangle .
\end{aligned}
$$

It follows that

$$
\begin{aligned}
\left\|x_{n+1}-x^{*}\right\|^{2} & \leq\left[1-\alpha_{n}(\tau-\gamma \alpha)\right]\left\|x_{n}-x^{*}\right\|^{2}+2 \alpha_{n}\left\langle\gamma f\left(x^{*}\right)-\mu \bar{F} x^{*}, j\left(x_{n+1}-x^{*}\right)\right\rangle \\
& \leq\left(1-\mu_{n}\right)\left\|x_{n}-x^{*}\right\|^{2}+\mu_{n} \delta_{n},
\end{aligned}
$$

where $\mu_{n}=\alpha_{n}(\tau-\gamma \alpha)$ and $\delta_{n}=\frac{2}{\tau-\gamma \alpha}\left\langle\gamma f\left(x^{*}\right)-\mu \bar{F} x^{*}, j\left(x_{n+1}-x^{*}\right)\right\rangle$. It is easy to see that $\sum_{n=1}^{\infty} \mu_{n}=\infty$ and $\limsup _{n \rightarrow \infty} \delta_{n} \leq 0$. Hence, by Lemma 2.3 , the sequence $\left\{x_{n}\right\}$ converges strongly to $x^{*} \in \operatorname{Fix}(T)$. From $x^{*}=\lim _{t \rightarrow 0} x_{t}$ and Theorem 3.1, we have that $x^{*}$ is the unique solution of the variational inequality $\left\langle(\mu \bar{F}-\gamma f) x^{*}, j\left(x^{*}-u\right)\right\rangle \leq 0, u \in \operatorname{Fix}(T)$.

On the other hand, suppose that $x_{n} \rightarrow x^{*} \in \operatorname{Fix}(T)$ as $n \rightarrow \infty$, where $x^{*}$ is the unique solution of the variational inequality $\left\langle(\mu \bar{F}-\gamma f) x^{*}, j\left(x^{*}-u\right)\right\rangle \leq 0, u \in \operatorname{Fix}(T)$. Observe that

$$
\begin{aligned}
\left\|\alpha_{n}\left(\gamma f\left(x_{n}\right)-\mu \bar{F} T x_{n}\right)\right\| & =\left\|x_{n+1}-T x_{n}\right\| \\
& \leq\left\|x_{n+1}-x^{*}\right\|+\left\|T x^{*}-T x_{n}\right\| \\
& \leq\left\|x_{n+1}-x^{*}\right\|+\left\|x_{n}-x^{*}\right\| \rightarrow 0 \quad(n \rightarrow \infty) .
\end{aligned}
$$

This completes the proof.

Remark 3.4 Compared with Theorem 3.2 of Tian [7], our Theorem 3.3 improves and extends Theorem 3.1 of Tian [7] in the following aspects:

(i) The framework of a Hilbert space is extended to a uniformly convex and 2-uniformly smooth Banach space.

(ii) The $\eta$-strongly monotone operator $F$ is extended to the case of an $\eta$-strongly accretive operator $\bar{F}$.

(iii) We establish a necessary and sufficient condition for the strong convergence of nonexpansive mappings. It follows from (C1) that $\alpha_{n}\left(\gamma f\left(x_{n}\right)-\mu \bar{F} T x_{n}\right) \rightarrow 0$ $(n \rightarrow \infty)$. Hence, we can obtain Theorem 3.2 of Tian [7] immediately. Thus, our Theorem 3.3 covers Theorem 3.1 of Tian [7] as a special case. 
The following example shows that all the conditions of Theorem 3.3 are satisfied. However, condition (C1) is not satisfied.

Example 3.5 Let $X=R$ be the set of real numbers. Define the mappings $T: X \rightarrow X, f$ : $X \rightarrow X$ and $\bar{F}: X \rightarrow X$ as follows:

$$
T x=0, \quad \bar{F} x=x \quad \text { and } \quad f(x)=\frac{1}{2} x \quad \forall x \in R .
$$

It is easy to see that $K=\frac{\sqrt{2}}{2}, \alpha=\frac{1}{2}$ and $\operatorname{Fix}(T)=\{0\}$. By $\bar{F} x=x$, we have $\eta=k=1$ and hence $0<\mu \leq \eta /\left(2 k^{2} K^{2}\right)=1$. Also, put $\mu=1$. It is easy to see that $\tau=1-\sqrt{1-2 \mu \eta+2 \mu^{2} k^{2} K^{2}}=1$. From $0<\gamma \alpha<\tau$, we have $\gamma \in(0,2)$. Without loss of generality, we put $\gamma=1$. Given sequences $\left\{\alpha_{n}\right\}$ and $\left\{\sigma_{n}\right\}, \alpha_{n}=1 / 2, o\left(\alpha_{n+1}\right)=1 / n^{2}$ and $\sigma_{n}=0$ for all $n \geq 0$. For an arbitrary $x_{0} \in X$, let $\left\{x_{n}\right\}$ be defined as

$$
x_{n+1}=\left(I-\alpha_{n} \mu \bar{F}\right) T x_{n}+\alpha_{n} \gamma f\left(x_{n}\right), \quad n \geq 0,
$$

that is,

$$
x_{n+1}=\frac{1}{2} \cdot \frac{1}{2} x_{n}=\frac{1}{4} x_{n}, \quad n \geq 0 .
$$

Observe that for all $n \geq 0$,

$$
\left\|x_{n+1}-0\right\|=\frac{1}{4}\left\|x_{n}-0\right\|
$$

Hence we have $\left\|x_{n+1}-0\right\|=\left(\frac{1}{4}\right)^{n+1}\left\|x_{0}-0\right\|$ for all $n \geq 0$. This implies that $\left\{x_{n}\right\}$ converges strongly to $0 \in \operatorname{Fix}(T)$.

Observe that $\langle(\mu \bar{F}-\gamma f) 0, j(0-u)\rangle \leq 0, u \in \operatorname{Fix}(T)$, that is, 0 is the solution of the variational inequality $\left\langle(\mu \bar{F}-\gamma f) x^{*}, j\left(x^{*}-u\right)\right\rangle \leq 0, u \in \operatorname{Fix}(T)$.

Finally, we have

$$
\left\|\alpha_{n}\left(\gamma f\left(x_{n}\right)-\mu \bar{F} T x_{n}\right)\right\|=\left\|\frac{1}{2}\left(\frac{1}{2} x_{n}-0\right)\right\|=\frac{1}{4}\left\|x_{n}\right\| \rightarrow 0 \quad(n \rightarrow \infty) .
$$

Hence there is no doubt that all the conditions of Theorem 3.3 are satisfied. Since $\alpha_{n}=$ $1 / 2$, condition (C1): $\lim _{n \rightarrow \infty} \alpha_{n}=0$ of Tian [7] is not satisfied.

Competing interests

The authors declare that they have no competing interests.

Authors' contributions

Both authors contributed equally and significantly to this research article. Both authors read and approved the final manuscript.

\section{Author details}

${ }^{1}$ School of Mathematical Sciences, Soochow University, Suzhou, 215006, China. ${ }^{2}$ School of Mathematical Sciences, Yancheng Teachers University, Yancheng, 224051, China.

\section{Acknowledgements}

The authors would like to thank the referees for giving useful suggestions and comments for the improvement of this paper. This study was supported by the Natural Science Foundation of Jiangsu Province under Grant (13KJB110028), and the National Science Foundation of China (11271277). 


\section{References}

1. Takahashi, W: Nonlinear Functional Analysis. Yokohama Publishers, Yokohama (2000)

2. Goebel, K, Kirk, WA: Topics on Metric Fixed-Point Theory. Cambridge University Press, Cambridge (1990)

3. Takahashi, Y, Hashimoto, K, Kato, M: On sharp uniform convexity, smoothness, and strong type, cotype inequalities. J. Nonlinear Convex Anal. 3, 267-281 (2002)

4. Yamada, I: The hybrid steepest descent for the variational inequality problems over the intersection of fixed points sets of nonexpansive mapping. In: Butnariu, D, Censor, Y, Reich, S (eds.) Inherently Parallel Algorithms in Feasibility and Optimization and Their Application, pp. 473-504. Elsevier, New York (2001)

5. Lions, PL: Approximation de points fixes de contractions. C. R. Acad. Sci. Paris Sér. A-B 284, 1357-1359 (1977)

6. Xu, HK, Kim, TH: Convergence of hybrid steepest-descent methods for variational inequalities. J. Optim. Theory Appl. $119,185-201(2003)$

7. Tian, M: A general iterative algorithm for nonexpansive mappings in Hilbert spaces. Nonlinear Anal. 73, 689-694 (2010)

8. Xu, HK: Inequalities in Banach spaces with applications. Nonlinear Anal. 16, 1127-1138 (1991)

9. Liu, LS: Iterative processes with errors for nonlinear strongly accretive mappings in Banach spaces. J. Math. Anal. Appl. 194, 114-125 (1995)

10. Xu, HK: Iterative algorithms for nonlinear operators. J. Lond. Math. Soc. 66, 240-256 (2002)

11. Deimling, K: Zero of accretive operators. Manuscr. Math. 13, 365-374 (1974)

doi:10.1186/1687-1812-2014-106

Cite this article as: Wang and Qian: A necessary and sufficient condition for the strong convergence of nonexpansive mappings in Banach spaces. Fixed Point Theory and Applications 2014 2014:106.

\section{Submit your manuscript to a SpringerOpen ${ }^{\circ}$ journal and benefit from:}

- Convenient online submission

- Rigorous peer review

- Immediate publication on acceptance

Open access: articles freely available online

High visibility within the field

- Retaining the copyright to your article 